\title{
A Gait Generation for Biped Robot Based on Artificial Neural Network and Improved Self-Adaptive Differential Evolution Algorithm
}

\author{
Van-Tinh Nguyen, Tam Bui, and Hiroshi Hasegawa
}

\begin{abstract}
Gait generation is very important as it directly effects on the quality of locomotion of biped robots. In point of mathematical view, a gait generation problem is considered as an optimization problem with constraints, it is readily engaged itself to Evolutionary Computation methods and solutions. This paper proposes a novel approach for gait pattern generation of the biped robot. This is to aim to enable the robot to walk more naturally and more stably in locomotion on flat ground. In this study, the approximated optimization method based on Artificial Neural Networks (ANNs) and Improved Self-Adaptive Differential Evolution Algorithm (ISADE) for a gait generation problem is mentioned. To evaluate the achievement of the proposed method, the robot was simulated by multi-body dynamic simulation software, Adams (MSC software, USA). Besides, the walking behavior of the robot is also considered in comparison with that of the human. The result shows that the new approach is an effective method for a gait generation of the biped robot.
\end{abstract}

Index Terms-ANNs, biped robot, ISADE, gait generation.

\section{INTRODUCTION}

Human has a complicated physical structure and implements difficult movements. During the past several decades, many researchers in the world have concentrated on the field of the biped robot inspired by humans [1]-[5]. The first aim of researches carried out in this field attempts to solve the following problem: How can the robot walk naturally and stably. This goal is motivated by several applications of the biped robot development such assistance, entertainment and medical issues. Hence, they have to move in a domestic environment and should have the same ability as humans to carry out stable walking.

To reach this target, several works were done for the generation of the walk of biped robot using the reinforcement learning [6], [7]. Furthermore, a big majority of the walk pattern researches was done based on the zero moment point (ZMP) criterion. Likewise, Kondo et al. [8] described an algorithm for emulations of disabled person's gait based on the ZMP criterion. Also, a constrained analytical trajectory filter [9] was a part of an analytical motion filter using the zero moment point as the stability criteria.

Some works of the walking gaits were based on

Manuscript received September 15, 2016; revised December 28, 2016. Van-Tinh Nguyen is with Shibaura Institute of Technology, Japan (e-mail: mf15422@shibaura-it.ac.jp).

Tam Bui is with Hanoi University of Science and Technology, Vietnam. Hiroshi Hasegawa is with Shibaura Institute of Technology, Japan developing the passive dynamics walking and using other methods such as central pattern generator (CPG) [10]. Also, Narukawa et al. [11] focused on the use of passive dynamics to achieve efficiently walking with simple mechanisms by using a numerical approach.

On the other hand, several researchers consider gait generation as a multiconstrained, multiobjective optimization problem. Gait generation, which combines to control the robot's gait. For instance, human motion captured data has been collected to drive a humanoid robot [12]. However, a number of papers indicate that biological locomotion data can not be used directly for a biped robot caused by kinematic and dynamic discrepancies between humans and the biped robot. This indicates the need for kinematic adjustments in calculating joint angle trajectory [12].

The second strategy considers the gait generation problem of the biped robot as an optimization problem with constraints. The optimal gait cycle is generated by minimizing some performance indexes, for instance, the velocity of motion [13], [14], stability criteria, energy consumption [15], [16], and so on. The gait generation problem of the biped robot often has several objectives and some of the objectives may be inconsistent to each other (for example speed and stability). Hence, it can be said that the gait generation problem is a multi-constrained and multi-objective optimization problem [17]. These two gait generation strategies may reach the same goal by different methods since both of them actually solve the gait synthesis problem as a multi-constrained multi-objective optimization problem. This paper addresses to a novel approach for gait generation based on combining two above-mentioned strategies. It is called approximated optimization method using ANNs and ISADE [18]. From human motion captured data, gait functions are interpolated. After that, the optimization problem is formulated to optimize the coefficients of gait function. ANNs is applied to approximate objective function and constraint functions. In this paper, ISADE once again performs an outstanding approach to multi-constraints, multi-peak problems. It was capable of attaining robustness, high quality, low calculation outstanding efficient performance on various problems in comparison to standard Differential Evolution (DE) algorithm. In addition, in this study, a new foot structure with toes is also proposed for the biped robot. The rest of this paper is organized into five sections. Section II describes the mechanical structure of the biped robot. The optimization procedure is in Section III. Section IV describes the methodology. Section V shows the results of the experiment confirmed by Adams. Finally, Section VI includes some 
conclusions.

\section{EXPERIMENTAL ROBOT MODEL}

\section{A. Overview of Structural Design}

In this study, the proposed model is built based on the KHR-3HV robot of Kondo Kagaku Company [19] which is the third generation of a humanoid robot developed by this company. The KHR-3HV robot has the weight of $1.5 \mathrm{~kg}$, the height of $401.05 \mathrm{~mm}$ and up to 22 Degree of Freedom (DOF) with 17 actual servos and 5 dummy servos. However, in this work, robot legs are concentrated. Thus, upper body joints are fixed and lower body have 10 controlled joints for the legs as shown in Fig. 1.

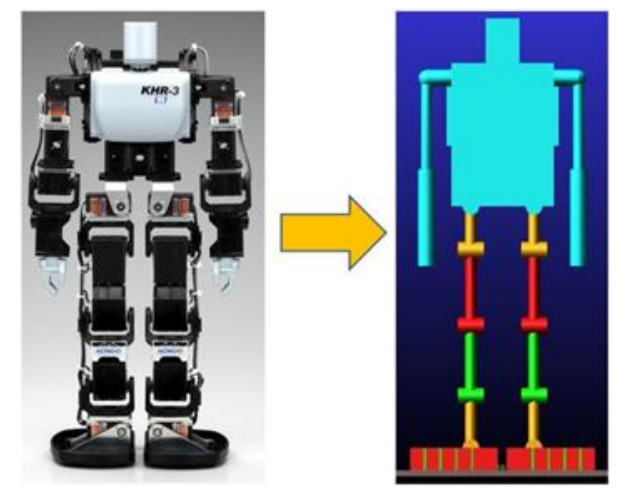

Fig. 1. Real robot and simulation model.

\section{B. Foot Mechanism}

During a stance phase of the human walk, support area continuously varies on the sole of the foot as described in Fig. 2. At the end of toe-off, there is a phase switch from stance to swing. Toes have an important role in this process, it makes the phase switch smoother and more stable due to a decrease of the effect of ground reaction force varied unexpectedly.

By this idea, a new foot structure with toes is proposed to enhance the walking behavior of the biped robot as depicted in Fig. 3.

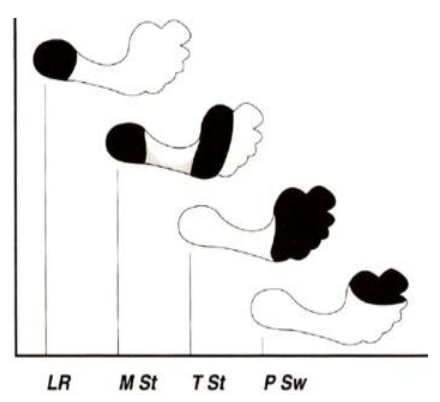

Fig. 2. Sequence of foot support areas During stance phase [20].
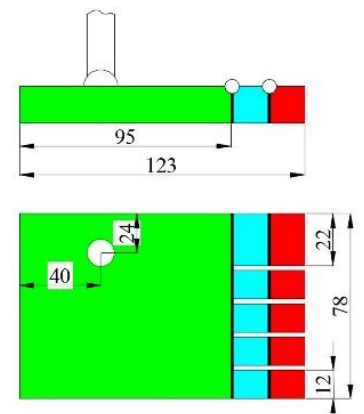

Fig. 3. Foot structure with toes.
Fig. 3 shows the proposed foot basic dimensions. In [21], Chockalingam et al. have proved that average ratio between the foot length and heel varies from 1.196 to 1.426 . Thus, the length of heel and foot are designed of $95 \mathrm{~mm}$ and $123 \mathrm{~mm}$. The ankle joint position is determined based on the real robot.

As proven in [22], the biped robot whose big toe ratio per foot width equals 0.28 , has the longest walking distance when big toe length is fixed and this ratio is similar to the ratio of the human foot. Therefore, the width of big toe and foot are designed of $22 \mathrm{~mm}$ and $78 \mathrm{~mm}$. Since KHR-3HV is a small biped robot, energy saver is noticeable. In toe mechanism, considering a reduction in energy consumption, the passive joint using torsion spring was selected as a toe joint. Spring stiffness coefficients are respectively set of $0.52 \mathrm{~N} . \mathrm{mm} / \mathrm{deg}$, $0.26 \mathrm{~N} . \mathrm{mm} / \mathrm{deg}$ to big toe joint and lesser toe joints. Coefficients of static and kinetic friction are 0.5, 0.417 for heel and ground, $0.17,0.155$ for toe and ground, respectively.

\section{OPTIMIZATION PROCEDURE}

\section{A. Definition of Joint Angle}

The joint angles are defined as described in Fig. 4. The range of angle is determined based on human motion data as in Table I.
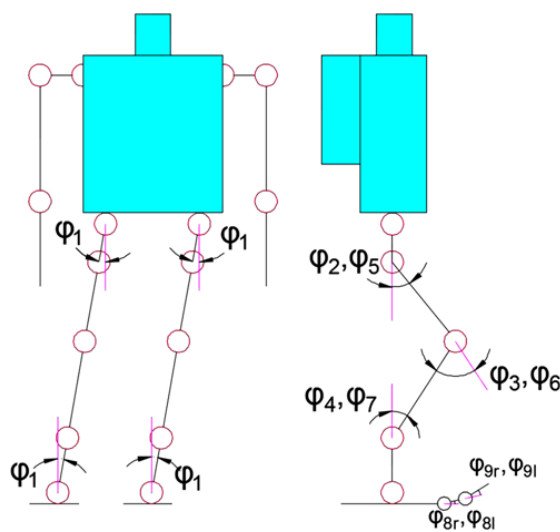

Fig. 4. Robot linkage model.

TABLE I: THE RANGE OF JOINT ANGLE

\begin{tabular}{cllll}
\hline \hline Angle & View plane & Leg & \multicolumn{1}{c}{ Joint } & \multicolumn{1}{c}{ Value } \\
\hline$\varphi_{1}$ & Frontal & Both & Hip and ankle & $-15^{\circ}$ to $15^{\circ}$ \\
$\varphi_{2}$ & Sagittal & Right & Hip & $-50^{\circ}$ to $50^{\circ}$ \\
$\varphi_{3}$ & Sagittal & Right & Knee & $0^{\circ}$ to $60^{\circ}$ \\
$\varphi_{4}$ & Sagittal & Right & Ankle & $-50^{\circ}$ to $50^{\circ}$ \\
$\varphi_{5}$ & Sagittal & Left & Hip & $-50^{\circ}$ to $50^{\circ}$ \\
$\varphi_{6}$ & Sagittal & Left & Knee & $0^{\circ}$ to $60^{\circ}$ \\
$\varphi_{7}$ & Sagittal & Left & Ankle & $-50^{\circ}$ to $50^{\circ}$ \\
$\varphi_{8 \mathrm{r}}$ & Sagittal & Right & Proximal phalanx & $0^{\circ}$ to $30^{\circ}$ \\
$\varphi_{9 \mathrm{r}}$ & Sagittal & Right & Distal phalanx & $0^{\circ}$ to $30^{\circ}$ \\
$\varphi_{81}$ & Sagittal & Left & Proximal phalanx & $0^{\circ}$ to $30^{\circ}$ \\
$\varphi_{91}$ & Sagittal & Left & Distal phalanx & $0^{\circ}$ to $30^{\circ}$ \\
\hline \hline
\end{tabular}

\section{B. Gait function}

Basing on the human walking pattern as depicted in [23], suppose that the robot control data was generated by the gait function as trigonometric function shown in Equation 1.

$$
\varphi_{i}(t)=a_{i}+b_{i \cdot} \cdot \cos (\omega t)+c_{i} \cdot \sin (\omega t)+b_{i} \cdot \cos (\omega t)
$$

where $\varphi_{i}$ is the angle of $i$ joint, $a, b, c, d$ are coefficients, $\mathrm{t}$ is time, and $\omega$ is angular velocity. By changing $a, b, c, d$ coefficients, the gait function will be created to allocate to each joint of the robot.

In this study, the biped robot is considered the locomotion on flat ground with the total time of 4.8 seconds. The robot is simulated in 3 cycles which spend on 3.6 seconds, 1.2 left seconds is used for checking robot stability. One cycle is set up to 1.2 seconds. As a result, the angular velocity is calculated by Equation 2. In the simulation, one step takes 
0.02 second, the total number of step is 240 . In the second cycle, the biped robot performs its motion the most natural, hence this cycle will be selected to show the waveform of gait function.

$$
\omega=\frac{2 . \Pi}{1.2}=5.236(\mathrm{rad} / \mathrm{s})
$$

Gait functions are assigned to all joints as Equation 3-9.

$$
\begin{gathered}
\varphi_{1}=\left\{\begin{array}{r}
0, t=0 \text { or } t \geq 3.6 \\
\pm 1.5, t=0.3 \text { and } t=3.3 \\
\varphi_{1}(t), 0.3<t<3.3
\end{array}\right. \\
\varphi_{2}=\left\{\begin{array}{r}
0, t \leq 0.3 \text { or } t \geq 3.6 \\
\varphi_{2}(t+0.6), 0.3<t<3.3 \\
15, t=3.3
\end{array}\right. \\
\varphi_{3}=\left\{\begin{array}{rr}
0, t \leq 0.3 \text { or } t \geq 3.6 \\
\varphi_{3}(t+0.6), 0.3<t<3.3 \\
30, t=3.3
\end{array}\right. \\
\varphi_{4}=\left\{\begin{array}{r}
0, t \leq 0.3 \text { or } t \geq 3.6 \\
\varphi_{4}(t+0.6), 0.3<t<3.3 \\
15, t=3.3
\end{array}\right. \\
\varphi_{5}=\left\{\begin{array}{rr}
0, & t=0 \text { or } t \geq 3.3 \\
15, & t=0.3 \\
\varphi_{2}(t), & 0.3<t<3.3
\end{array}\right. \\
\varphi_{6}=\left\{\begin{aligned}
0, & t=0 \text { or } t \geq 3.3 \\
30, & t=0.3 \\
\varphi_{3}(t), & 0.3<t<3.3
\end{aligned}\right. \\
\varphi_{7}=\left\{\begin{array}{rr}
0, & t=0 \text { or } t \geq 3.3 \\
15, & t=0.3 \\
\varphi_{4}(t), & 0.3<t<3.3
\end{array}\right.
\end{gathered}
$$

In toe mechanism, due to considering a reduction in the energy consumption of the robot, the passive joint is selected as toe joint. Consequently, $\varphi_{8 \mathrm{r}}, \varphi_{81}, \varphi_{9 \mathrm{r}}$, and $\varphi_{91}$ have values in the range from $0^{\circ}$ to $30^{\circ}$. Their values depend on the robot geometric posture as well as impact force when the robot walks.

\section{Problem Formulation}

The concept of the optimization process is shown as in Fig. 5. $Z_{f}$ and $X_{f}$ denote the distance from the initial position to final position along $\mathrm{Z}$-axis and $\mathrm{X}$-axis in the robot locomotion, respectively. $R_{f}$ is the angle of rotation.

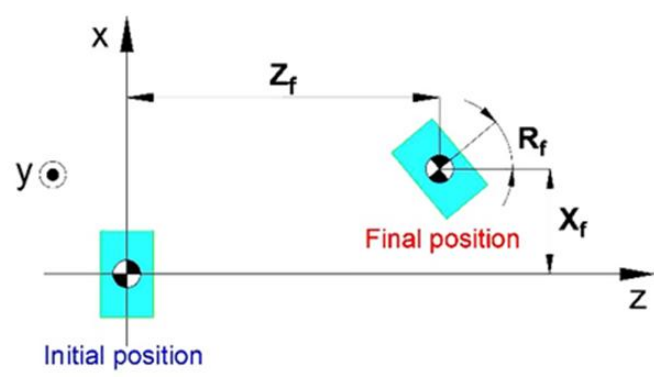

Fig. 5. Overview of optimization.

Definition of optimal design is described as Equation 10-17.

Design variables (DVs):

$$
x=\left[a_{i}, b_{i}, c_{i}, d_{i}\right], i=1 \div 4
$$

Constraint functions:

$$
\begin{aligned}
& g_{1}(x)=20-\left|X_{f}\right| \geq 0 \\
& g_{2}(x)=5-\left|R_{f}\right| \geq 0 \\
& g_{3}(x)=243.53-Y_{f} \leq 0 \\
& h(x)=N-240=0
\end{aligned}
$$

where $Y_{f}$ is distance from Centre of Mass (CoM) to ground. $N$ is a total simulation step.

Objective function:

$$
f(x)=-Z_{\mathrm{f}} \rightarrow \min
$$

Penalty function:

$$
P(x)=\sum_{i=1}^{3} \max \left(g_{i}(x), 0\right)+h(x)
$$

Modified objective function:

$$
F(x)=-Z_{\mathrm{f}}+\gamma \cdot P \rightarrow \min
$$

where $a_{i}, b_{i}, c_{i}, d_{i}(i=1,2,3,4)$ are the coefficients of gait function. There are four constraint functions. In Equation 11-12, $X_{f}$ distance and $R_{f}$ angle are constrained under $\pm 20 \mathrm{~mm}$ and $\pm 5^{\circ}$ to ensure that the biped robot can walk straight. In Equation 13, $Y_{f}$ must be more than $243.53 \mathrm{~mm}$ to ensure the robot not to slip and fall down at the final framework. In Equation 14, N is equal to 240 to check the success of the simulation. In Equation 17, $\gamma$ is a penalty coefficient set to 1000. Equation 11-14 will be also checked again when the simulation finishes.

\section{METHODOLOGY APPROACH}

\section{A. Neural Network Overview}

Neural network (NN) is a mathematical model that aims to represent certain characteristics of brain functions. This study was based on models of the living brain. Models of the brain are becoming increasingly significant due to advances in neuroscience, especially the distinction between biology and neuroscience (also known as ANNs). NNs were first modeled by McCulloch and Pitts in 1943. The learning method was proposed by Hebb in 1949, which forms the basis of the current network learning method. The perception neural network was proposed in 1958.

The neural network can be divided into three major learning paradigms such as supervised learning, unsupervised learning, and reinforcement learning. If supervised learning is that you are provided a mapping implied by the data, unsupervised learning is used for data clustering. As a result of any reduced dimension, for linearly inseparable problems and the amount of multi-dimensional data such as images and statistics, a good solution is relatively obtained with a small amount of calculation. From this fact, including data mining and pattern recognition, have been applied in various fields.

Back-propagation is a method used by the three-layer structure found in most NNs. Fig. 6 shows the output of each neuron out $_{j}$, a sum of weights net $_{j}$, the output unit connection weights from the hidden units $w_{j k}$ and the connection weights to the hidden units from the input layer unit $w_{j i}$. The 
back-propagation error activation function (a sigmoid function) described in Equation 20 is often used. To determine whether the signal of output layer is much closer to the teacher signal, the error back-propagation method defines the squared error E, which can be expressed using Equation 21. Thus, if E approaches 0, the signal of the output layer approaches the teacher signal. Therefore, the purpose of the back-propagation method is to determine the weight of $w_{j i}$, $w_{j k}$.

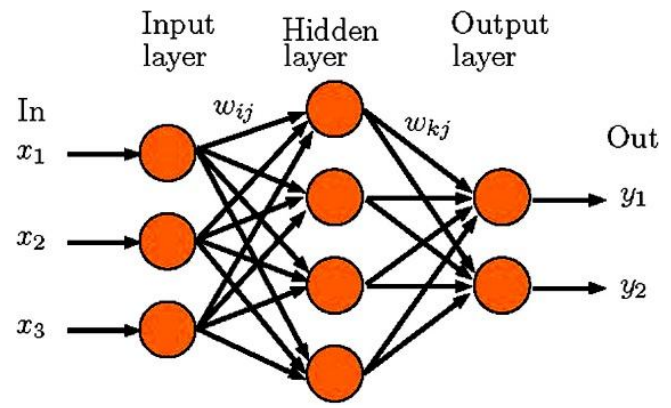

Fig. 6. ANN architecture.

$$
\begin{gathered}
n e t_{j}=\sum_{i=0}^{L n} w_{j i} . \text { out }_{i} \\
w_{j} n^{n} i^{n}=y_{n m} / y_{(n-1) m} \\
f\left(\text { net }_{j}\right)=1 /\left(1+\exp ^{-n e t_{j}}\right) \\
E=\frac{1}{2} \cdot \sum_{i}^{L n}\left(y_{p i}-\text { out }_{i}\right)^{2} ;(1 \leq p \leq P)
\end{gathered}
$$

\section{B. Improved Self-Adaptive Differential Evolution}

Differential evolution (DE) is an optimization technique originally proposed by Storn and Price [24]. It is categorized into evolution algorithm group, which is characterized by operators of mutation and crossover. In DE, two important coefficients, which play key roles to decide the correction and speed of convergence, are scaling factor $\mathrm{F}$ and crossover rate $\mathrm{C}_{\mathrm{r}}$. Another important parameter in $\mathrm{DE}$, population size $\mathrm{NP}$ remains a user-assigned value to cope with problem complexity. ISADE not only adaptively changes those three coefficients but also integrate different mutation schemes to take advantages of them.

\section{1) Adaptive learning strategies selection}

In [18], Tam Bui et al. randomly chose three mutation schemes, which are DE/best/1/bin, DE/best/2/bin, and DE/rand best/1/bin. Among DE's schemes, DE/best/1/bin and $D E /$ best/2/bin are known for good convergence property and $D E /$ rand best/1/bin is known for good diversity. The probability of applying those strategies are equally assigned at with values $p_{1}=p_{2}=p_{3}=1 / 3$. Equation 8 shows the formula of chosen schemes.

$$
\begin{gathered}
\text { DE/best/1: } V_{i, j}^{G}=X_{\text {best }, j}^{G}+F .\left(X_{r 1, j}^{G}-X_{r 2, j}^{G}\right) \\
\text { DE/best/2: } V_{i, j}^{G}=X_{\text {best }, j}^{G}+F .\left(X_{r 1, j}^{G}-X_{r 2, j}^{G}\right)+ \\
F .\left(X_{r 3, j}^{G}-X_{r 4, j}^{G}\right) \\
\text { DE/best/2: } V_{i, j}^{G}=X_{\text {best }, j}^{G}+F .\left(X_{\text {best }, j}^{G}-X_{r 2, j}^{G}\right)+ \\
F .\left(X_{r 3, j}^{G}-X_{r 4, j}^{G}\right)
\end{gathered}
$$

In APGA/VNC approach proposed by S. Tooyama and H.
Hasegawa [25] scaling factor changes according to iteration as sigmoid function as in Equation 23.

$$
F_{i}=\frac{1}{1+\exp \left(\alpha \frac{\left.i-\frac{N P}{N P}\right)}{N}\right.}
$$

ISADE give addition scaling $F_{i}^{\text {mean }}$ as in Equation 24 .

$$
F_{i}^{\text {mean }}=\mathrm{F}_{\min }+\left(\mathrm{F}_{\max }-\mathrm{F}_{\min }\right)\left(\frac{i_{\max }-i}{i_{\max }}\right)^{n_{\text {iter }}}
$$

where

$$
n_{i t e r}=n_{\min }+\left(n_{\max }-n_{\min }\right) \frac{i}{i_{\max }}
$$

$F_{i}$ in Equation 23 is modified as in Equation 26

$$
F_{i}=\frac{F_{i}+F_{i}^{\text {mean }}}{2}
$$

Now scaling factor is set to be high in the first iteration and after certain generations, it becomes smaller for proper exploitation.

\section{2) Crossover control parameter}

ISADE algorithm is able to detect whether high values of $\mathrm{C}_{\mathrm{r}}$ are useful and if a rotationally invariant crossover is required. A minimum base for $\mathrm{C}_{\mathrm{r}}$ around its median value is incorporated to avoid stagnation around a single value. The control parameter $C_{r}$ is assigned as Equation 27.

$$
C_{r}^{i+1}=\left\{\begin{aligned}
\text { rand }_{2} & \text { if } \text { rand }_{1}<\tau \\
C_{r}^{i} & \text { otherwise }
\end{aligned}\right.
$$

where $\operatorname{rand}_{1}$ and $\operatorname{rand}_{2}$ are random values $\in[0,1]$, $\tau$ presents probability to adjust $C_{r}$. $C_{r}$ is adjusted as in Equation 28 .

$$
C_{r}^{i+1}= \begin{cases}C_{r_{\min }} & \text { if } C_{r_{\text {min }}} \leq C_{r}^{i+1} \leq C_{r_{\text {medium }}} \\ C_{r_{\max }} & \text { if } C_{r_{\text {medium }}} \leq C_{r}^{i+1} \leq C_{r_{\max }}\end{cases}
$$

where $C_{r_{\text {min }}}, C_{r_{\text {medium }}}, C_{r_{\text {max }}}$ denote low value, median value and high value of crossover parameter respectively. As in [18], we take $\tau=0.1, C_{r_{\text {min }}}=0.05, C_{r_{\text {medium }}}=0.5$, $C_{r_{\text {max }}}=0.95$.

All above ideas and theories are implemented as in flowchart in Fig. 7.

\section{Optimization Solution}

The optimization process is implemented as the following steps:

Step 1. Setting up ranges for variables and design of experiment based on random samples.

Step 2. To collect and filter sample data by simple analysis.

Step 3. Building the architecture of ANN for approximating an objective function and constraint functions.

Step 4. The objective function approximated by ANN is optimized by using ISADE.

Step 5. Design variables from step 4 are used to check constraint functions again through the simulation.

Step 6. The convergence is checked. If this is achieved, the optimal process will be terminated. Oppositely, the repetition will begin from step 4 . 


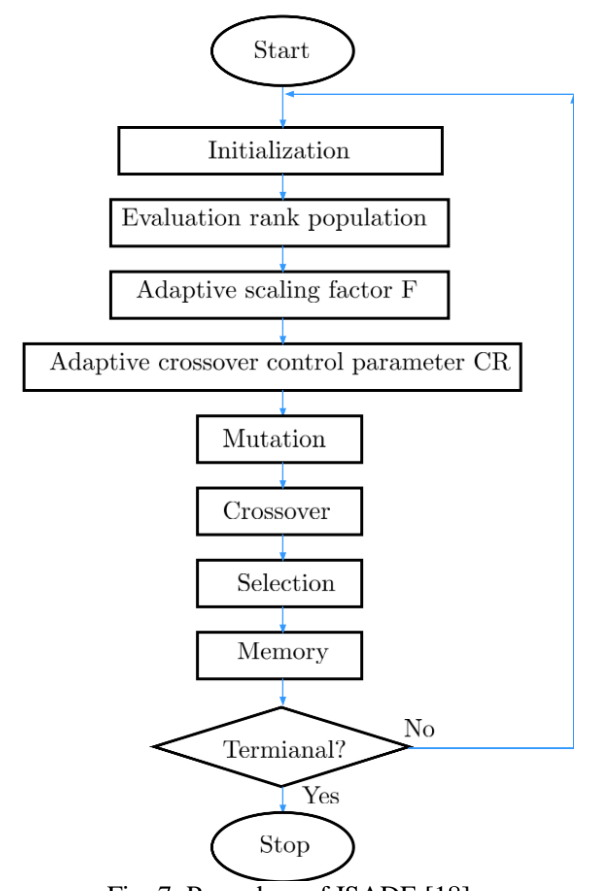

Fig. 7. Procedure of ISADE [18].

\section{EXPERIMENT AND RESULT}

\section{A. Data Fitting}

To determine the weights of $w_{j i}$. $w_{j k}$, the total number of sample for training ANN is 200. This number of the experiment is decided based on the response surface model $3^{\text {rd }}$ order as described in Equation 29. Some more experiments are implemented to enhance the accuracy of the approximated model.

$$
N_{s}=\frac{(N+1)(N+2)}{2}+N
$$

where $N$ is the number of design variables, $N=16$. The approximation is shown as in Fig. 8. The accuracy of the method is evaluated by R-squared analysis which is more than $0.95(95 \%)$. This value performs the success of the approximation model.

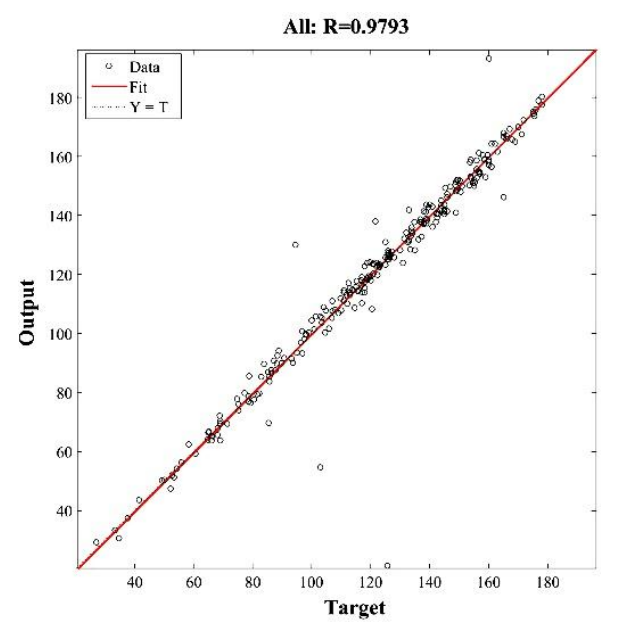

Fig. 8. Experimental and predicted values of ANN model.

\section{B. Simulation Result}

In the optimization process using ISADE algorithm, we set the number of iteration to 1000 or the fitness function value does not increase in 50 consecutive iterations. The results are described as in Table II and Fig. 9. F8 performs the best result with $X_{f}, Z_{f}, R_{f}$ of $0.044 \mathrm{~mm}, 177.96 \mathrm{~mm}$ and $1.2^{\circ}$, respectively.

In [26], Michael S Orendurff et al. have examined the differences in limb kinematics and kinetics, which occur while walking straight ahead and around $1 \mathrm{~m}$ radius circular path at constant speed. When walking straight ahead, the CoM oscillates between foot contacts in a sinusoidal pattern as depicted in Fig. 10. In comparison with the human, the robot's CoM shown in Fig. 11 also has a sinusoidal wave and the foot trajectories have a curve tread like a human foot trajectory. The difference mainly appears due to physical structure dissimilarity.

\begin{tabular}{ccccc}
\multicolumn{5}{c}{ TABLE II: SimUlation RESULT } \\
\hline \hline$N_{\mathrm{o}}$ & $X_{f}(\mathrm{~mm})$ & $Z_{f}(\mathrm{~mm})$ & $R_{f}($ degree $)$ & $N$ \\
\hline$F_{1}$ & 0.725 & 154.56 & 3.837 & 240 \\
$F_{2}$ & 2.962 & 160.22 & -0.431 & 240 \\
$F_{3}$ & 0.837 & 161.78 & -2.291 & 240 \\
$F_{4}$ & 19.690 & 165.50 & 4.419 & 240 \\
$F_{5}$ & -6.273 & 165.74 & -4.201 & 240 \\
$F_{6}$ & 5.380 & 166.34 & 1.139 & 240 \\
$F_{7}$ & -4.770 & 168.30 & 0.796 & 240 \\
$F_{8}$ & 0.044 & 177.96 & 1.203 & 240 \\
\hline \hline
\end{tabular}

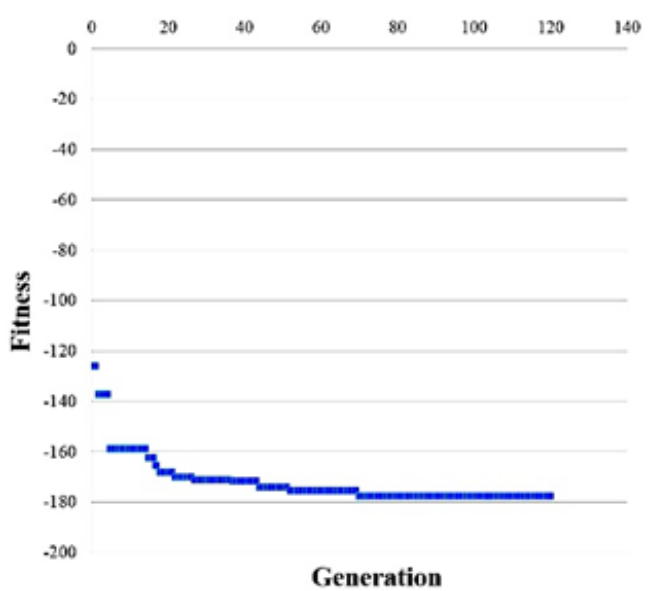

Fig. 9. Optimization process convergence.

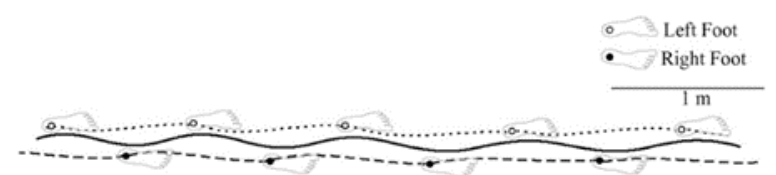

Fig. 10. Human's CoM trajectory during straight ahead walking [26].

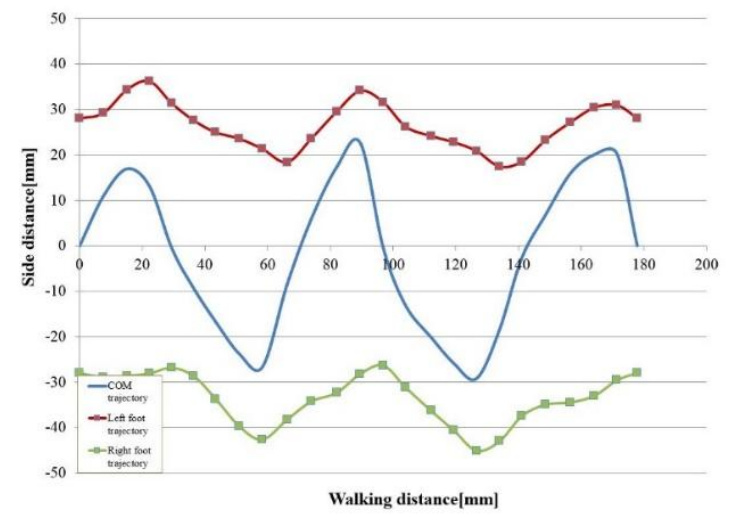

Fig. 11. CoM and ankle position trajectory in locomotion on flat ground.

A consideration of the gait similarity between the robot 
and the human in locomotion on flat ground is depicted as in Fig. 12. The robot gait at time 1.2s likes an "Initial contact period" of the human gait, at time $1.5 \mathrm{~s}$ likes "Midstance period", at time 1.8s likes "Terminal stance period", at time $2.1 \mathrm{~s}$ likes "Initial swing period" where it can be observed the bending of toes and at time $2.4 \mathrm{~s}$ likes "Terminal swing period". The differences of posture are expected to occur as a consequence of the physical structure dissimilarity with the human's structure. The waveform of gait function allocated to all joints of the robot is shown in Fig. 13. As can be seen, the waveforms of hip and knee joint gait function are similar to that of human beings.

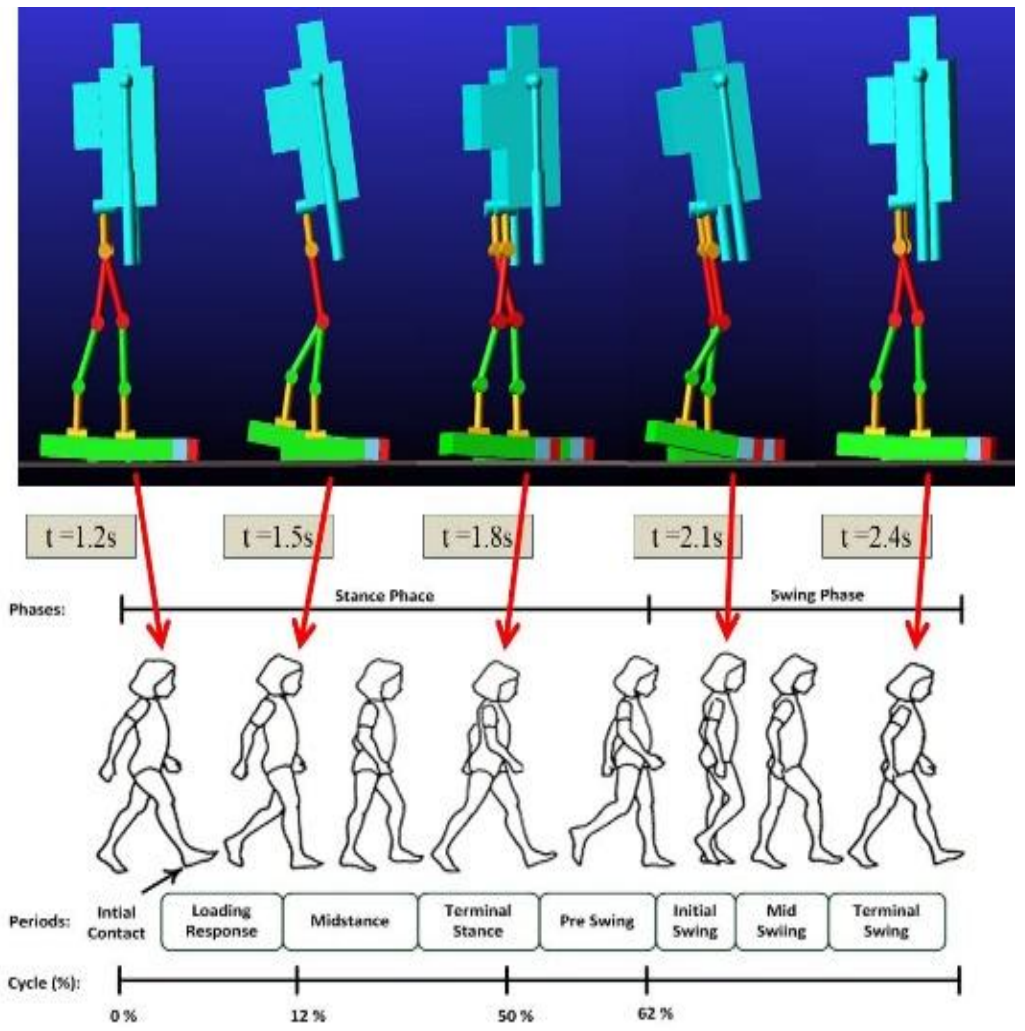

Fig. 12. Gait comparison of the biped robot with the human.

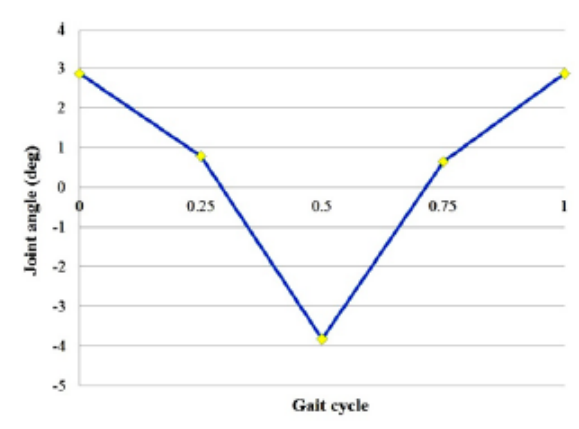

Fig. 13a. A cycle of gait function (hip and ankle roll joint angle)

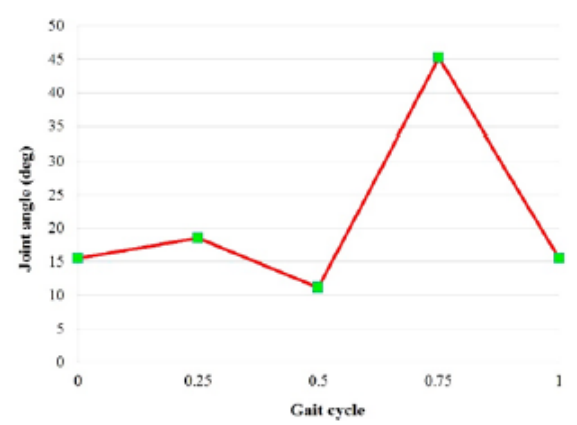

Fig. 13c. A cycle of gait function (knee pitch joint angle)

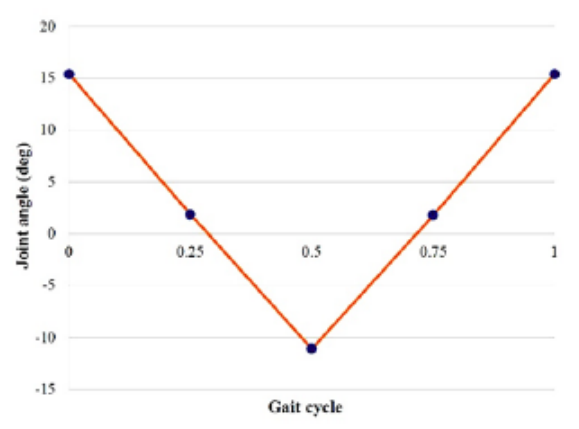

Fig. 13b. A cycle of gait function (hip pitch joint angle)

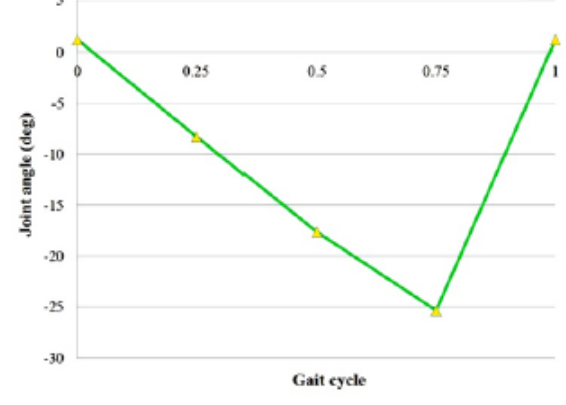

Fig. 13d. A cycle of gait function (ankle pitch joint angle)

Fig. 13. Waveform of the gait function.

\section{CONCLUSION}

A gait pattern generation is a very challenging task in the biped robot area. Recently, the approach of using evolutionary algorithms (EAs), especially new methods, proved their potential of tackling gait generation problem based on their effectiveness on searching for optimal result. 
In this paper, to generate walking gait of a small biped robot, a novel gait pattern generation method is proposed called the approximated optimization method based on ISADE and ANN. Through the dynamic simulation of the robot locomotion by Adam software, the result showed that the novel approach has high performance for a gait pattern generation problem. ANN one again performs the excellent predictive capabilities for the non-linear problem. With ISADE algorithm as a searching tool, it is not necessary to have good initials to avoid local minima and converge to near-global minimal solutions. ISADE using self-adaptive coefficients shows its effective for a multiconstrained problem with fast convergence and accuracy. Besides, the proposed approach reduced the complexity of gait pattern generation problem in comparison to the conventional methods mentioned in previous works. Finally, this study plans to do improvement with an objective function such as energy cost.

\section{ACKNOWLEDGMENT}

This work was supported by Hybrid Twinning Program of Shibaura Institute of Technology, Japan. I would like to express my heartfelt thanks to Shibaura Institute of Technology, especially Prof. Hiroshi Hasegawa for his suggestion and help in this study.

\section{REFERENCES}

[1] Y. Sakagami, R. Watanabe, C. Aoyama, S. Matsunaga, N. Higaki, and K. Fujimura, "The intelligent ASIMO, system overview and integration," in Proc. the IEEE/RSJ Int. Conf. on Intelligent Robot and Systems, 2002, pp. 2478-2483.

[2] S. Lohmeier, K. Loffler, M. Gienger, H. Ulbrich, and F. Pfeiffler, "Computer system and control of biped Johnnie," in Proc. IEEE Int. Conf. on Robotics and Automation, vol. 4, 2004, pp. 4222-4227.

[3] Y. Ogura, H. Aikawa, K. Shimomura, A. Morishima, H. O. Lim, and A. Takanishi, "Development of a new humanoid robot WABIAN-2," in Proc. IEEE Int. Conf. on Robotics and Automation, 2006, pp. 830-835.

[4] K. Kaneko, F. Kanehiro, S. Kajita, K. Yokoyama, K. Akachi, T. Kawasaki, S. Ota, and T. Isozumi, "Design of prototype humanoid robotics platform for HRP," in Proc. the IEEE/RSJ Int. Conf. on Intelligent Robots and Systems, 2002, pp. 2431-2436.

[5] T. Ishida, "Development of small biped entertainment robot QRIO," in Proc. The Fourth Symposium Micro-Nano mechatronics for Information-Based Society, 2004, pp. 23-28.

[6] L. Yang, C. M. Chew, Y. Zheng, and A. N. Poo, "Truncated Fourier series formulation for bipedal walking balance control," Robotica, vol. 28, pp. 81-96, 2010.

[7] T. Li, Y. T. Su, S. W. Lai, and J. J. Hu, "Walking motion generation, synthesis, and control for biped robot by using PGRL, LPI, and fuzzy logic," IEEE Trans. on Systems, Man, and Cybernetics, Part B: Cybernetics, vol. 41, pp. 736-748, 2011.

[8] H. Kondo, Y. Ogura, H. Aikawa, A. Morishima, J. Shimizu, H. Lim, and A. Takanishi, "Application of biped humanoid robot to simulate the motion of elderly and disable people," Gerontechnology, vol. 7, p. 143.

[9] K. Muecke and D. Hong, "Constrained analytical trajectory filter for stabilizing humanoid robot motions," Intelligent Service Robotics, vol. 4, pp. 203-218, 2011.

[10] C. Fu, F. Tan, K. Chen, “A simple walking strategy for biped walking based on an intermittent sinusoidal oscillator," Robotica, vol. 28, pp. 869-884, 2010

[11] T. Narukawa, M. Takahashi, and K. Yoshida, "Efficient walking with optimization for a planar biped walker with torso by hip actuators and springs," Robotica, vol. 29, pp. 641-648, 2011.

[12] C. Zhou, P. K. Yue, J. Ni, and S. B. Chan, "Dynamically stable gait planning for a humanoid robot to climb sloping surface," in Proc. IEEE Int. Conf. on Robotics, Automation and Mechatronics, pp. 341-346, 2004.

[13] M. Aghaabbasloo, M. Azarkaman, and M. E. Salehi, "Biped robot joint trajectory generation using PSO evolutionary algorithm," in Proc. $3^{\text {rd }}$
Joint Conf. of Al \& Robotic and $5^{\text {th }}$ RoboCup Iran Open Int. Symp. 2013, pp. 1-6.

[14] P. H. Kuo, Y. F. Ho, K. F. Lee, L. H. Tai, and T. H. S. Li, "Development of humanoid robot simulator for gait learning by using particle swarm optimization," in Proc. IEEE Int. Conf. on Systems, Man, and Cybernetics, 2013, pp. 2683-2688.

[15] T. Arakawa and T. Fukuda, "Natual motion trajectory generation of biped locomotion robot using genetic algorithm through energy optimization," in Proc. IEEE Int. Conf. on Systems, Man, and Cybernetics, vol. 2, 1996, pp. 1495-1500.

[16] L. Hu, C. Zhou, and Z. Sun, "Estimating biped gait using spline-based probability distribution function with Q-learning," IEEE Trans. on Industrial Electronics, vol. 55, pp. 1444-1452, 2008.

[17] K. Seo and S. Hyun, "Genetic programming based automatic gait generation for quadruped robots," in Proc. the $10^{\text {th }}$ Annual Genetic and Evolutionary Computation Conference, 2008, pp. 293-294.

[18] T. Bui, H. Pham, and H. Hasegawa, "Improve self-adaptive control parameters in differential evolution for solving constrained engineering optimization problems," Journal of Computational Science and Technology, vol. 7, no. 1, pp. 59-74, 2013.

[19] KHR-3HV biped robot. [Online]. Available: http://kondo-robot.com/product/khr-3hv-ver-2-life

[20] J. Perry and J. M. Burnfield, Gait Analysis: Normal and Pathological Function, $2^{\text {nd }}$ ed., SLACK Incorporated, 2010, ch. 4, p. 82.

[21] N. Chockalingam and R. Ashford, "Selected foot length ratios in non-pathological sample," Revista Internacional de Ciencias Podológicas, vol. 1, no. 2, pp. 25-30, 2007.

[22] K. Nerakae and H. Hasegawa, "Big toe sizing design of small biped robot by using gait generation method," Applied Mechanics and Materials, vols. 541-542, pp. 1079-1086, 2014.

[23] M. W. Whittle, An Introduction to Gait Analysis, $4^{\text {th }}$ ed., Oxford, 2007, ch. 2, p. 59.

[24] R. Storn and K. Price, "Differential Evolution-A simple and efficient heuristic for global optimization over continuous spaces," Journal Global Optimization, vol. 11, pp. 341-359, 1997.

[25] S. Tooyama and H. Hasegawa, "Adaptive Plan System with Genetic Algorithm using the Variable Neighborhood Range Control," in Proc. IEEE Congress on Evolutionary Computation, 2009, pp. 846-853.

[26] M. S. Orendurff, A. D. Segal, J. S. Berge, K. C. Flick, D. Spanier, and G. K. Klute, "The kinematics and kinetics of turning: limb asymmetries associated with walking a circular path," Gait and Posture, vol. 23, pp. 106-111, 2006.

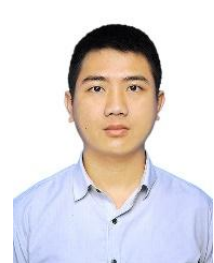

Van-Tinh Nguyen received his B.E. in 2012 at Hano University of Science and Technology (HUST), Vietnam and M.E. in 2016 from Shibaura Institute of Technology (SIT), Japan. Besides, he works as a lecturer at School of Mechanical Engineering of HUST. Currently, he is pursuing Dr. Eng. degree in Functional Control Systems at SIT. His research interests include optimization system design, multi-body systems, humanoid robot, and evolutionary algorithm.

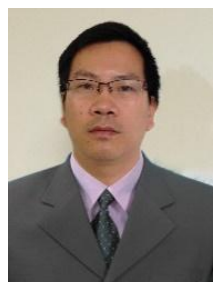

Tam Bui received the B.E. in 2008 at Hanoi University of Science and Technology, Vietnam. He received M.E. in 2012 and Dr. Eng. in 2015 in Functional Control Systems from Shibaura Institute of Technology, Japan. Currently, he is a lecturer at School of Mechanical Engineering, Hanoi University of Science and Technology, Vietnam. His research interests include optimization system design, biomimetic, swarm intelligent, evolutionary algorithm.

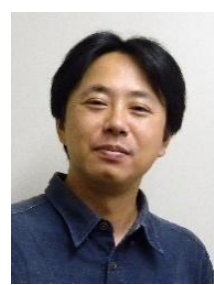

Hiroshi Hasegawa received his B.E. in 1992 and M.E. in 1994 from Shibaura Institute of Technology, Japan. He received Dr. Eng. In 1998 in mechanical engineering from Tokyo Institute of Technology, Japan. He has been working at Shibaura Institute of Technology, and currently is a Professor at the Department of Machinery and Control System, College of Systems Engineering and Science. He is a member of JSEE, JSME, ASME, JSCES, JSST and KES. His research interests include computer-aided exploration, creativity of design and systems engineering. 